\title{
Schizodeme analysis of Trypanosoma cruzi stocks from South and Central America by analysis of PCR-amplified minicircle variable region sequences
}

\author{
Herbert Avila ${ }^{1}$, Antonio M. Goncalves ${ }^{3}$, Nedia Saad \\ Nehme $^{3}$, Carlos M. Morel $^{3}$ and Larry Simpson ${ }^{1,2}$ \\ ${ }^{1}$ Department of Biology and ${ }^{2}$ Molecular Biology Institute, University of California, Los Angeles, CA, U.S.A \\ and ${ }^{3}$ Department of Biochemistry and Molecular Biology, Instituto Oswaldo Cruz, Rio de Janeiro, Brazil
}

(Received 5 February 1990; accepted 18 April 1990)

\begin{abstract}
Kinetoplast DNA (kDNA) was isolated from 56 stocks of Trypanosoma cruzi isolated from human patients, animals and insects from Brazil, Venezuela, Colombia and Costa Rica. Comparison of the patterns of digested kDNA on acrylamide gels led to the grouping of several stocks into two schizodemes. Schizodeme analysis was also performed using a set of 330-bp fragments representing all the variable regions of the minicircle DNA molecules, which were obtained by PCR amplification of the kDNA using conserved region primers. The results of this analysis were consistent with the analysis using total kDNA, but the more informative restriction profiles allowed the construction of additional schizodemes. In addition, two oligomers were generated from variable region sequences of cloned minicircles from a $\mathrm{Y}$ and a $\mathrm{Cl}$ strain, and these were used as schizodeme-specific probes to detect homologous sequences in the amplified minicircle DNAs. The results indicate that a combination of restriction enzyme fingerprinting and hybridization of amplified variable region minicircle DNA with schizodeme-specific probes can be used for both sensitive detection and classification of $T$. cruzi.
\end{abstract}

Key words: Trypanosoma cruzi; Polymerase chain reaction; Schizodeme; Minicircle DNA

\section{Introduction}

The kinetoplastid protozoan Trypanosoma cruzi is the causal agent of Chagas' disease, which is characterized by an acute phase after the initial infection and then a chronic phase in which there is a progressive degenerative disorder of the cardiac and/or gastrointestinal systems. Different isolates or stocks of $T$. cruzi exhibit a great deal of heterogeneity in terms of isoenzyme patterns, surface antigens, nuclear DNA content, morphology and kinetoplast minicircle DNA sequences. Differences in isoenzyme patterns and kinetoplast minicircle DNA sequences were used to distinguish related stocks or strains of $T$. cruzi which were operationally labeled 'zymodemes' [1] or 'schizodemes' [2], and theoretically defined [3,4] as non-sexually interacting clonal lines which

Correspondence address: L. Simpson, Biology Department, UCLA, Los Angeles, CA 90024-1606, U.S.A.

Abbreviations: kDNA, kinetoplast DNA; PCR, polymerase chain reaction. have been evolutionarily separated for a long time. Schizodeme analysis using total kinetoplast DNA (kDNA) has been shown to represent the method of choice for distinguishing different stocks and strains of this parasite [2]. However, this analysis requires at least $10^{8}$ cells, and it has been shown that the required outgrowth of parasites in insects, culture or animals may result in artificial selection of parasites from the original population present in the infected host $[5,6]$. We have shown previously that the polymerase chain reaction (PCR) can be used to amplify species-specific and possibly strain-specific fragments of minicircle DNA [7] from as few as 10 molecules, thereby allowing direct detection and classification of small numbers of parasites from infected patients or animals. In this paper we have established the generality of this method by analyzing 56 stocks of $T$. cruzi from different geographical areas of South and Central America, and have shown that the amplified variable region of the minicircle DNA is an appropriate species- and strain-specific marker for 
detection and classification of this parasite.

\section{Materials and Methods}

Trypanosoma cruzi stocks. Table I summarizes the host and geographical origin of the samples analyzed.

Isolation of total kDNA. Total kDNA was isolated as described previously $[2,8]$.

Schizodeme analysis of total kDNA. Total kDNA was digested with a restriction enzyme and the fragments separated by electrophoresis in $5.5-10 \%$ acrylamide gradient gels. The patterns were visualized by silver staining $[9,10]$.

Polymerase chain reaction amplification of minicircle sequences. Two pg of each kDNA sample were amplified in a $100 \mu l$ reaction using Taq DNA Polymerase (Perkin-Elmer Cetus). The reaction conditions were: $10 \mathrm{mM}$ Tris- $\mathrm{HCl} \mathrm{pH}$ $8.4 / 50 \mathrm{mM} \mathrm{KCl} / 6 \mathrm{mM} \mathrm{MgCl}_{2} / 0.01 \%$ gelatin/1 $\mathrm{mM}$ of each deoxytrinucleotide $/ 300 \mathrm{pmol}$ of each primer/2 units of Taq polymerase. $100 \mu \mathrm{l}$ of light mineral oil was layered on top of each reaction to prevent evaporation. The reactions were amplified for 35 cycles using a DNA Thermal Cycler (Perkin-Elmer Cetus). The cycling profile was as follows: $20 \mathrm{~s}$ denaturation at $94^{\circ} \mathrm{C}, 20 \mathrm{~s}$ annealing at $55^{\circ} \mathrm{C}$, and a $1 \mathrm{~min}$ elongation at $72^{\circ} \mathrm{C}$. An initial denaturation step of $4 \mathrm{~min}$ at $94^{\circ} \mathrm{C}$ and final elongation step of $10 \mathrm{~min}$ at $72^{\circ} \mathrm{C}$ were also included. A $5 \mu 1$ aliquot of each reaction was run on a $2 \%$ agarose gel to check the efficiency and specificity of the amplification reaction.

Schizodeme analysis of amplified minicircle kDNA. The amplified DNA was extracted once with chloroform to remove the mineral oil. The samples were then ethanol precipitated in the presence of $0.3 \mathrm{M}$ sodium acetate. The pellets were washed once with ethanol, vaccuum dried, and resuspended in $10 \mathrm{mM}$ Tris- $\mathrm{HCl} \mathrm{pH} 7.5 / 1 \mathrm{mM}$ EDTA. One third of each amplification reaction was used for a single restriction endonuclease digestion. Restriction enzymes were used according to the instructions of the manufacturer (Bethesda Research Laboratories). Digested samples were run in $5-15 \%$ gradient acrylamide gels in $1 \times$ Trisborate-EDTA electrophoresis buffer, at $100 \mathrm{~V}$ for $16 \mathrm{~h}$. The gels were stained in $0.5 \mu \mathrm{g} \mathrm{ml}^{-1}$ ethidium bromide for $30 \mathrm{~min}$ and photographed with $300 \mathrm{~nm}$ UV trans-illumination [11].

Oligonucleotides. Oligonucleotides were synthesized by standard phosphoramidite methods in an ABS DNA Synthesizer 381A. The PCR primers: P35: 5'-AAATAATGTACGGG(T/G)GAGATGCATGA- $3^{\prime}$ and

P36: 5'-GGGTTCGATTGGGGTTGGTGT-3' hybridize to the conserved region within the minirepeats of $T$. cruzi $\mathrm{kDNA}$ minicircles. The resulting amplification product is approximately 330 bp long and covers the adjacent variable region sequences in addition to a portion of the minirepeat [7]. The hybridization oligonucleotide probe:

P67: 5'-TGGTTTTGGGAGGGG(C/G)(G/C)(T/G)TCAA(A/C)TTT

hybridizes to the conserved region of the minirepeats. The oligonucleotide probes:

P173: 5'-TTACTTAATGAAAGTGTATCTG$\mathrm{AAG}-3^{\prime}$ and

P174: 5'-AGACGATACTCAGATTGTAGTAGAG-3'

were obtained from the variable regions of two cloned kDNA minicircles from a $\mathrm{Y}$ and a $\mathrm{Cl}$ strain, respectively [12].

Dot blot hybridizations. An aliquot of each amplification reaction containing the equivalent amount of DNA was denatured in $200 \mu \mathrm{l}$ of 0.4 $\mathrm{M} \mathrm{NaOH}, 25 \mathrm{mM}$ EDTA and applied to a $\mathrm{Ny}-$ tran (Schleicher and Schuell) filter. The DNA was fixed onto the membrane by cross-linking with short wavelength ultraviolet light. Hybridization conditions were: $6 \times$ SSC, $1 \times$ Denhardt's, $0.05 \%$ sodium pyrophosphate, $1 \%$ SDS and $20 \mu \mathrm{g} \mathrm{ml}^{-1}$ yeast tRNA. The oligonucleotide probes were $5^{\prime}$ end-labeled with $\left[{ }^{32} \mathrm{P}\right]$ ATP to a specific activity of $10^{9} \mathrm{cpm} \mu \mathrm{g}^{-1}$. The filters were hybridized at $45^{\circ} \mathrm{C}$ and then washed at $55^{\circ} \mathrm{C}$ (low stringency) or $65^{\circ} \mathrm{C}$ (high stringency) in $3 \mathrm{M}$ tetramethylammonium chloride solution [13] with 1\% SDS. 
TABLE I

Origin of T.cruzl kDNA samples

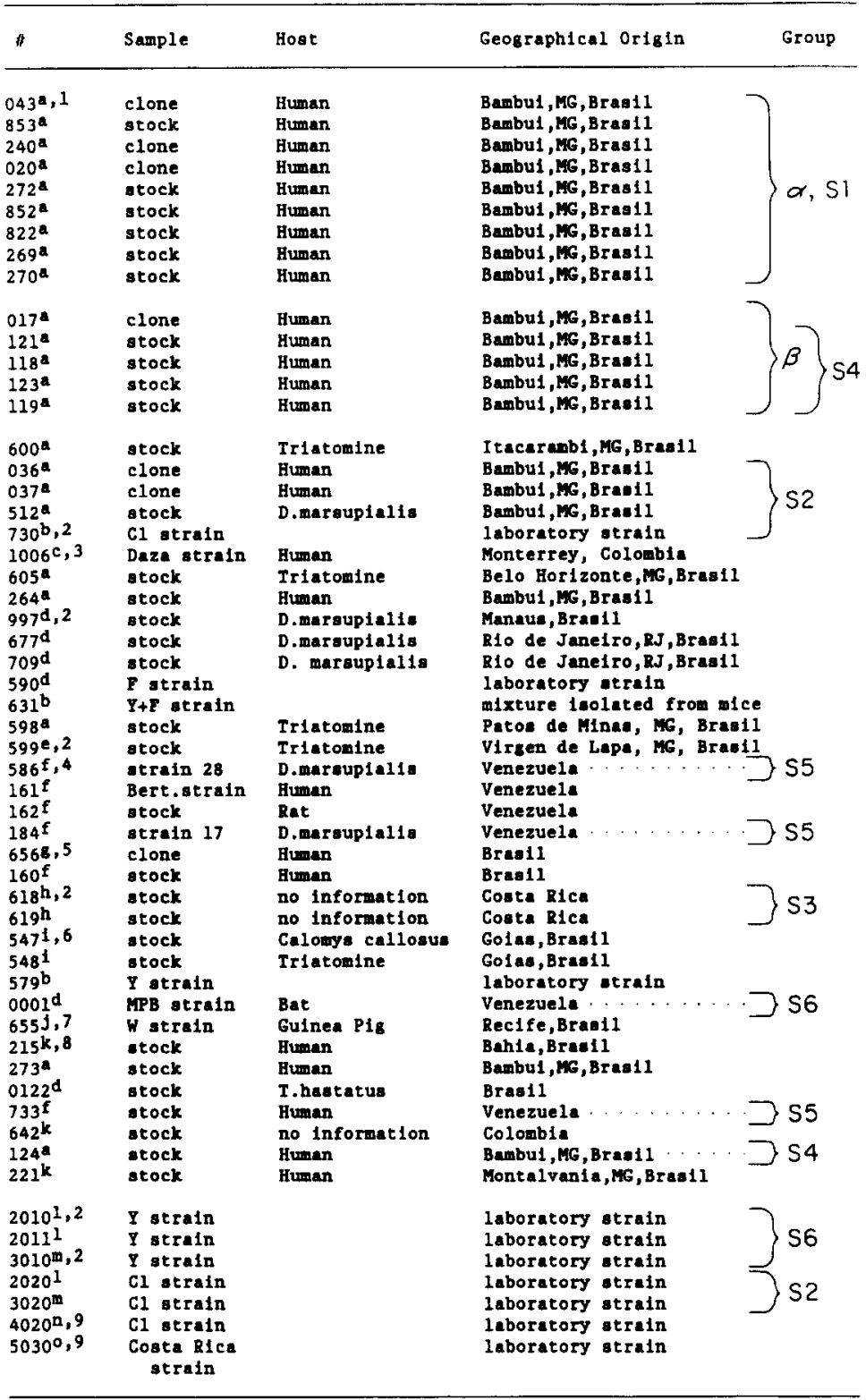

Samples were obtained frow the following laboratories:

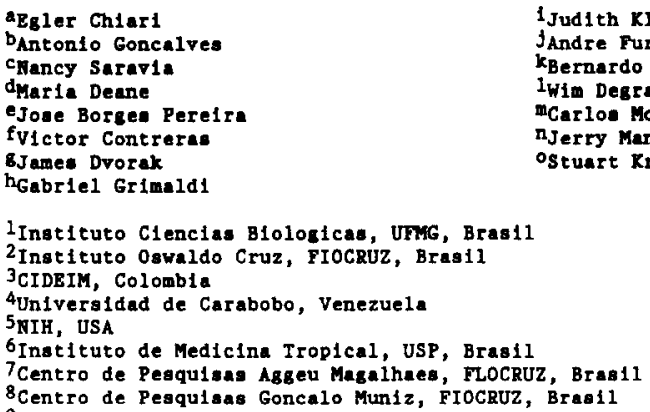

${ }^{8}$ Centro de Pesquias Goncalo Muniz, FIOCRUZ, Brasil

9Untversity of California at Irvine, USA 

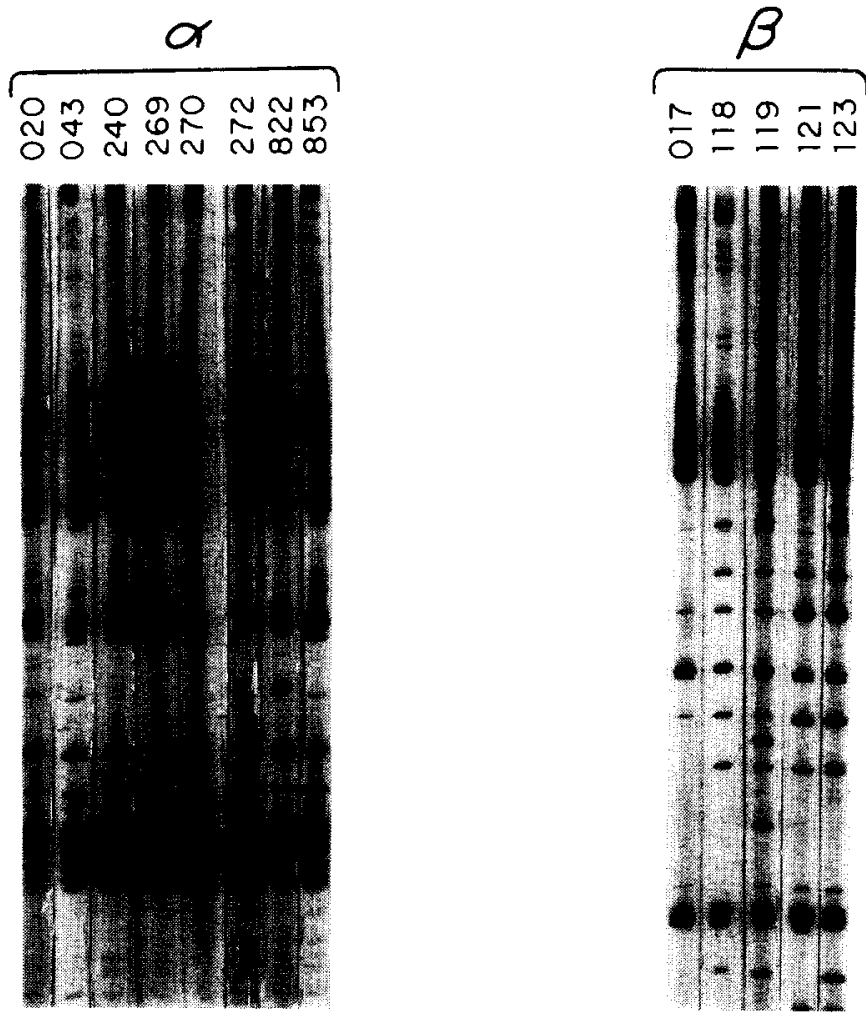

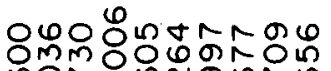

ำ

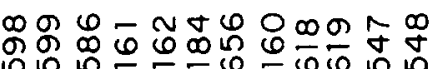

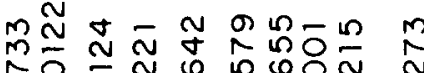

11"1"

1
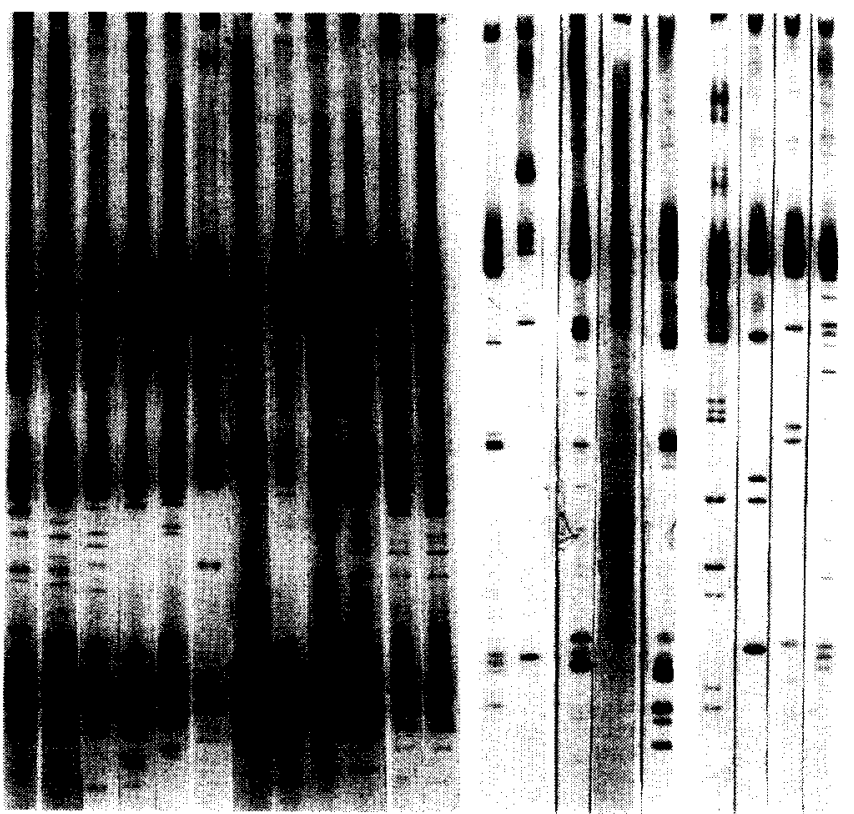

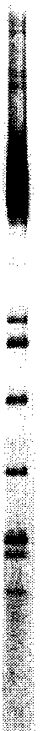




\section{Results}

Origins of T. cruzi kDNA samples. The origins of the $56 \mathrm{kDNA}$ samples are shown in Table I. Several of these samples are from cloned cell lines, but most are from uncloned stocks, and therefore may possibly consist of more than one strain. The parasites were isolated and cultured as epimastigotes from human patients, animals and insects from several regions of Brazil, Venezuela, Colombia and Costa Rica. The largest group of stocks came from Bambui, Brazil. Four laboratory stocks of $T$. cruzi which were labeled as $\mathrm{Cl}$ strain and four which were labeled as $Y$ strain were also analyzed. Sequences of several cloned kDNA minicircles from one of the $\mathrm{Cl}$ strains and one of the Y strains have been published [12].

Schizodeme analysis using digestion of total kDNA. Total kDNA was isolated from approximately $10^{9}$ epimastigote cells of each stock and digested with restriction enzymes. The EcoRI acrylamide gel profiles are shown in Fig. 1. Comparison of these patterns allowed the classification of the Bambui stocks into two schizodemes, the $\alpha$ - and $\beta$-groups. The remaining 34 samples are tentatively unclassified into schizodemes. However, it is clear by qualitative examination of the restriction profiles of the 34 unclassified stocks in Fig. 1 that these fall into three subgroups as shown. Further analysis is required to determine if these subgroups are valid. Samples 852, 037, 512 and the laboratory strains (except samples 730 and 590) were not analyzed in terms of total kDNA restriction fingerprints.

Polymerase chain reaction amplification of the $k D N A$ minicircle variable region from $56 T$. cruzi stocks. The P35/P36 primer set amplifies a 330 bp variable region fragment extending from one minirepeat region to the next in each kDNA minicircle molecule. This DNA amplification has been shown previously to be $T$. cruzi species-specific and, apparently, $T$. cruzi strain-independent [7]. The latter conclusion, however, was based only on the results from amplification of minicircle DNA from three $T$. cruzi laboratory stocks, which were labeled as the $\mathrm{Cl}, \mathrm{Y}$, and Peru strains.

In this paper we have analyzed an additional $54 T$. cruzi stocks and strains, plus the $\mathrm{Y}$ and $\mathrm{Cl}$ stocks previously tested. $2 \mathrm{pg}$ of total $\mathrm{kDNA}$, isolated from each of the $56 T$. cruzi stocks, was amplified using the P35/P36 primer set. The expected $330 \mathrm{bp}$ band was obtained for all 56 samples tested except one (0122), in which a slighter faster migrating product was obtained (Fig. 2). This indicates that the amplification of the variable region of the kDNA minicircle is probably general for all strains of $T$. cruzi.

Schizodeme analysis of the amplified variable region minicircle DNA. The 330-bp PCR products were digested with several restriction enzymes and the fragments separated in 5-15\% acrylamide gradient gels. The $R s a \mathrm{I}, T a q \mathrm{I}$ and HinfI restriction profiles are presented in Figs. 3-5. It is apparent that the two schizodemes obtained from analysis of total kDNA digests are also valid for the digestion patterns obtained with the 330-bp fragments. It is also apparent that the amount of information contained in the digestion patterns of the amplified 330-bp fragments is much greater than that contained in the total kDNA digestion patterns, where much of the information is obscured by the $1 / 4,2 / 4,3 / 4$ fragment pattern due to cleavage at one or more of the four conserved regions in the minicircle molecule. In order to differentiate the schizodeme classifications obtained using the amplified variable region minicircle DNA from those obtained using total kDNA, different designations are used. As shown in Table $I$, the kDNA schizodemes are indicated as $\alpha$ and $\beta$, whereas the amplified variable region schizodemes are indicated as S1-S6.

Fig. 1. Schizodeme analysis using total kDNA. Total kDNA from 46 of the samples listed in Table I was digested with EcoRI and the fragments separated in 5.5-10\% acrylamide gradient gels. The gels were silver stained. The samples are grouped according to the similarities of the gel patterns. Two schizodeme groups, $\alpha$ and $\beta$ (labeled 1 in Goncalves et al., 1990), are apparent. Samples 590 through 548 (grouped in the figure from left to right sequentially) were electrophoresed in separate gels from the other unclassified samples. 


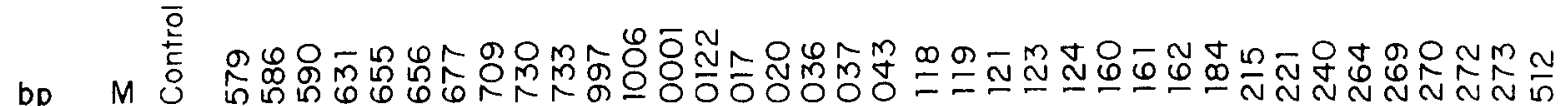

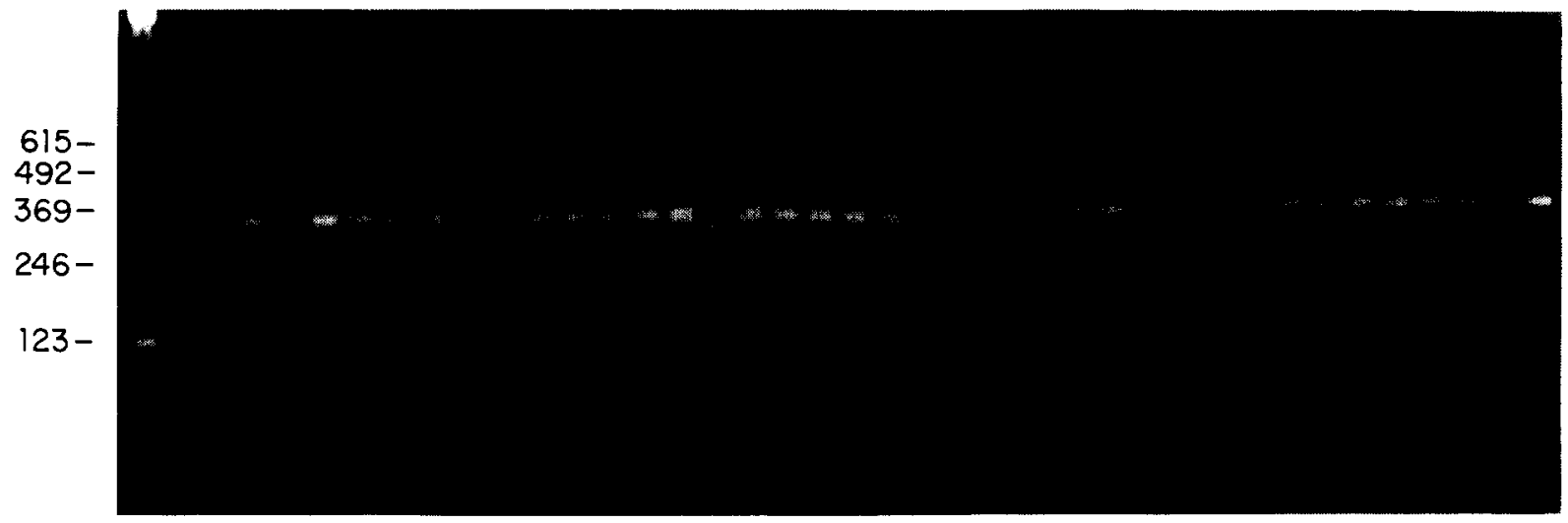

Fig. 2. Amplification of the 330-bp minicircle DNA variable region from $37 T$. cruzi stocks. All samples yielded the expected 330-bp PCR fragment except No. 122. Lane $M$ is a 123-bp ladder size marker. $2 \%$ agarose.

Schizodeme S1, which corresponds to the $\alpha$ schizodeme from total $\mathrm{kDNA}$, is clearly very homogeneous, as shown in Figs. 3A-5A. The use of several enzymes allowed a finer discrimination to be performed. For example, with $R$ saI digestion, all samples appeared very similar except for 822 . With TaqI digestion all samples, including 822, appeared similar. With HinfI digestion, samples 240,269 and 270 formed a related subgroup, and samples 272 and 852 formed another related subgroup. We conclude that it is important to compare digestion patterns obtained with several enzymes to make any firm deductions regarding the relatedness of various stocks.

Schizodeme S2 consists of stocks 036, 037, 512 and 730 . Since stock 730 was labeled a $\mathrm{Cl}$ laboratory strain, we also designate this the $\mathrm{Cl}$ schizodeme'. Three other putative $\mathrm{Cl}$ laboratory strains were also analyzed, but only two of them (2020 and 3020) were found to belong to the $\mathrm{Cl}$ schizodeme. Three of these stocks $(036,037$ and 512) were obtained from Bambui, Brazil. A schizodeme group not apparent with total kDNA, S5, consists of stocks 184,586 and possibly 733 . The relatedness of these three Venezuelan stocks, however, could only be visualized from the TaqI digestion patterns (Fig. 4A and 4C). The RsaI digestion patterns (Fig. 3C) suggested that two Costa Rican stocks 618 and 619 are related (= schizodeme S3). It should be noted that gel analysis of the stocks belonging to schizodeme S6 is not presented in Figs. 3-5, but is presented below in Fig. 6.

From total kDNA digestion patterns, the $\beta$ schizodeme is a relatively homogeneous subgroup (Fig. 1). However, comparison of the digestion patterns of the amplified DNA led to the conclusion that only four stocks within this schizodeme $-118,119,121$ and 123 - are very similar with all three enzymes (Figs. 3D-5D). In addition, it is possible that stock 124 , which was unclassified in Fig. 1, is also a member of this $\beta$ schizodeme. As is the case with all of the above well defined schizodemes, these stocks are derived from a single geographical area. In addition, a single geographical area can contain several schizodemes. For example, the stocks from Bambui, Brazil comprise three schizodemes $-\mathrm{S} 1$ (or $\alpha$ ), S2, and S4 (or $\beta$ ).

We conclude that restriction enzyme digestion of amplified minicircle variable region DNA provides more complex and richer profiles than digestion of total kDNA, allowing a more precise determination of the relatedness of $T$. cruzi strains. Digestion of total kDNA always contains the major $1 / 4,2 / 4$ and $3 / 4$ unit length bands which are derived from the conserved minirepeats, and this pattern often obscures the diagnostic complexities 


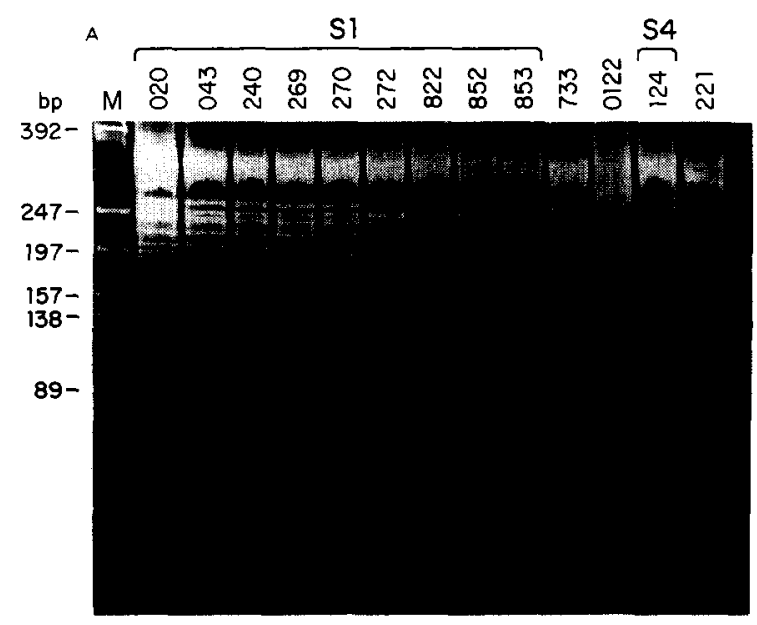

RsaI

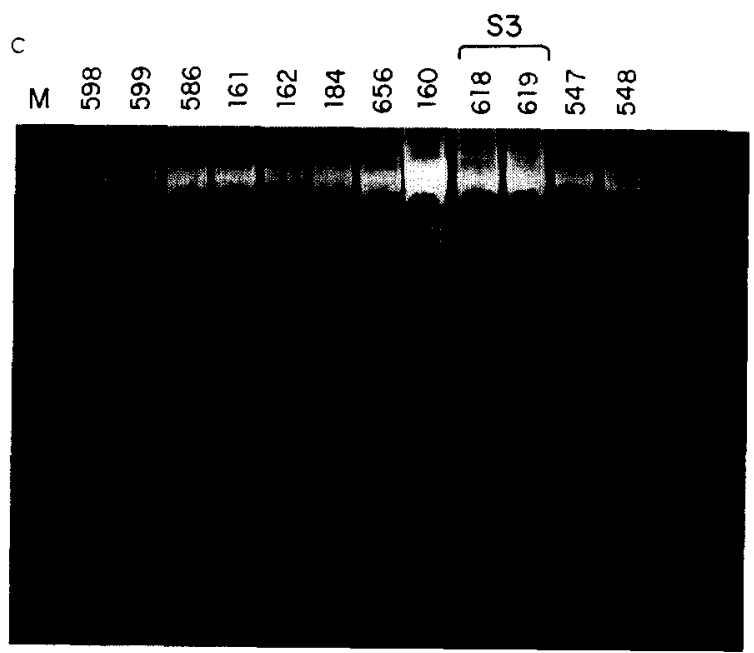

RsaI

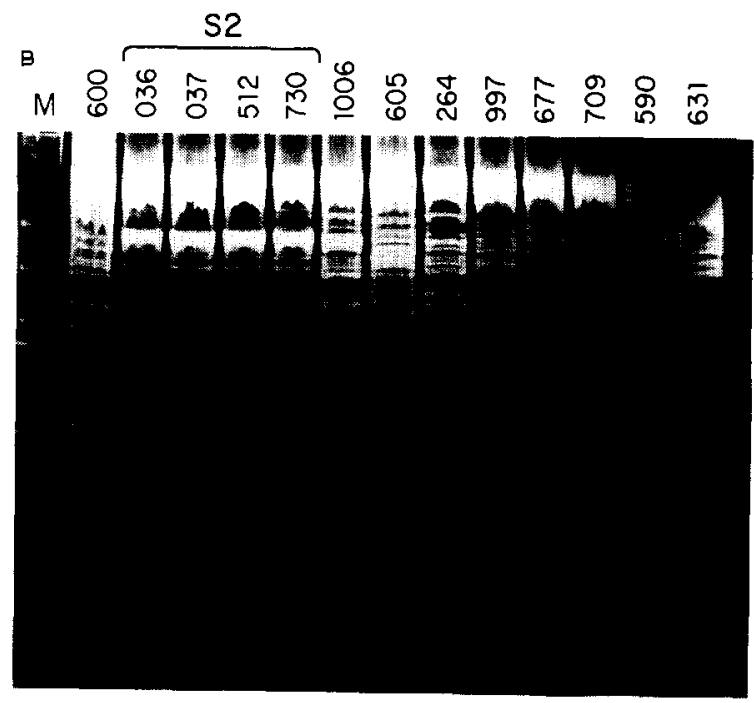

RsaI

D
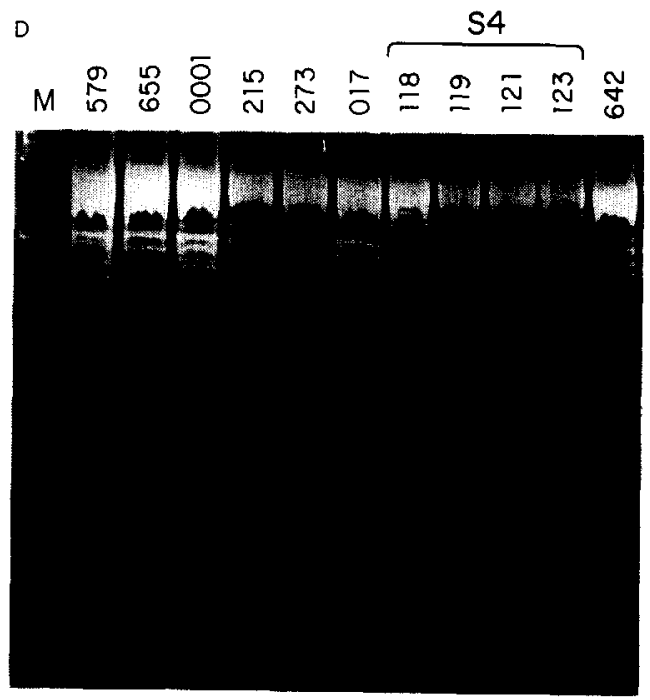

RsaI

Fig. 3. Schizodeme analysis of $T$. cruzi stocks by restriction digestion of PCR-amplified minicircle variable region DNA. The 330-bp PCR products from the stocks listed in Table I were digested with Rsal and the fragments separated on a 5-15\% acrylamide gradient gel. Gels were stained with ethidium bromide. The samples are grouped as in Fig. 1 for ease of comparison. Samples belonging to a particular schizodeme group are denoted by the schizodeme S number. Lane M, $\phi$ X174/RsaI size marker.

of the restriction patterns.

Dot blot hybridization with variable region probes for $\mathrm{Cl}$ and $\mathrm{Y}$ strains. We have shown previously that differences in patterns of kDNA digestion products indicate differences in sequences of minicircle fragments [2]. However, since a single network contains thousands of minicircles composed of $10-20$ different sequence classes as defined by the variable region sequences, the question must be addressed as to what is the actual variation in the frequency of different minicircle sequence classes between different schizodemes. If the variation is significant, then it might be possible to generate variable region hybridization probes 

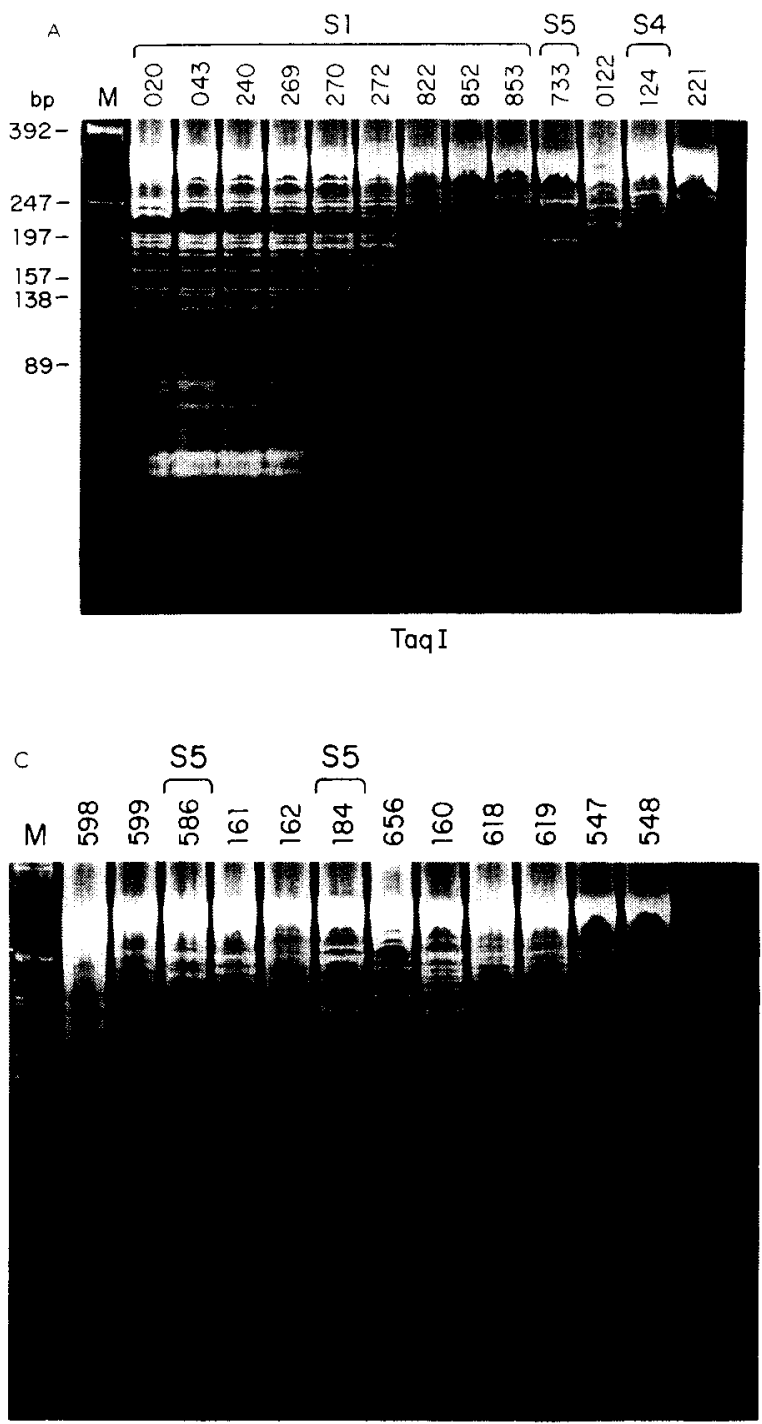

TaqI

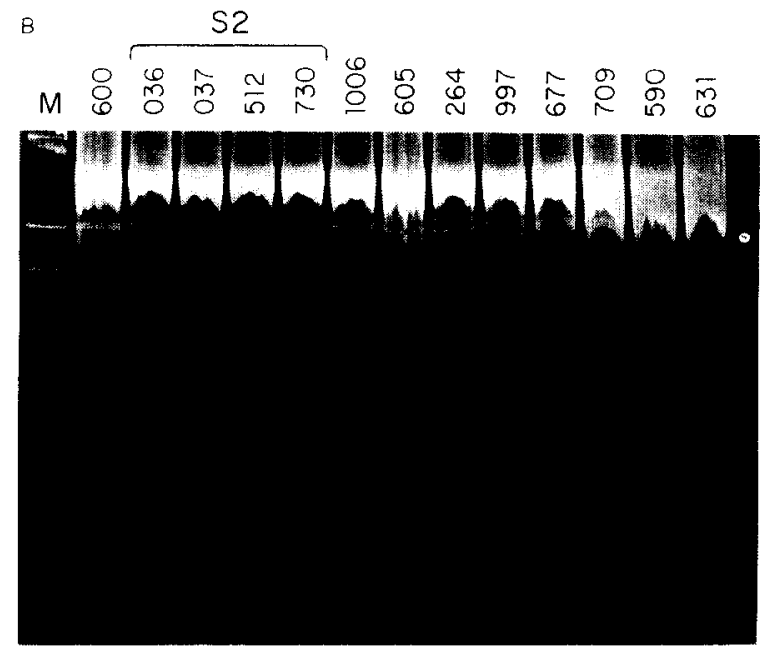

Taq I

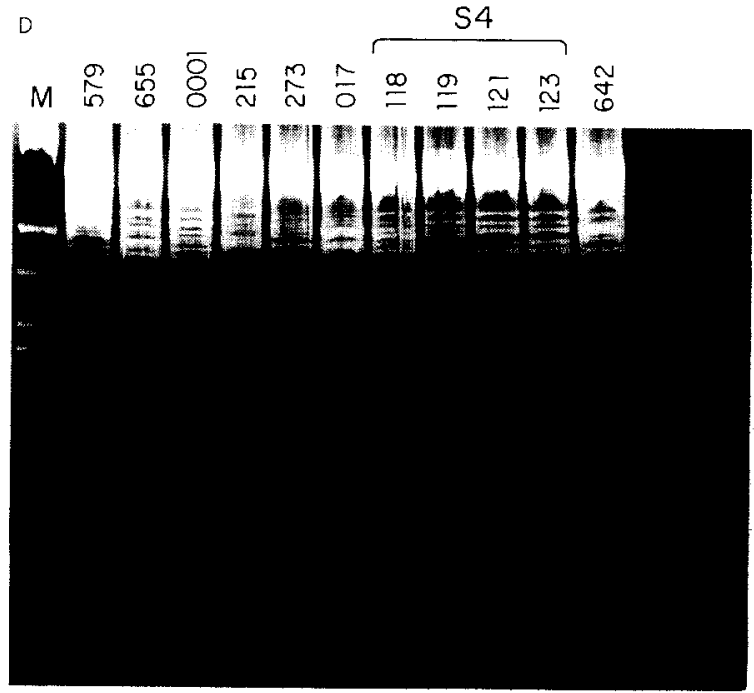

TaqI

Fig. 4. Schizodeme analysis of $T$. cruzi stocks by restrictıon digestion of PCR-amplified minicircle variable region DNA. The 330-bp PCR products from the stocks listed in Table I were digested with Taql. Conditions same as in Fig. 3. Lane M, $\phi \mathrm{X} 174 / R$ saI size marker.

which would be schizodeme-specific, and this might allow a rapid screening of amplified variable region minicircle DNA for the purpose of classification.

To answer this question, aliquots of the $330 \mathrm{bp}$ minicircle variable region amplification products for each of the 56 T. cruzi stocks were spotted onto filters. One filter was hybridized with an oligonucleotide probe (P67) complementary to the mini- circle conserved minirepeat region, in order to check for $T$. cruzi-specific minicircle DNA fragments: all samples showed positive hybridization (data not shown).

Replicate filters were hybridized with the oligonucleotide probes, P173 and P174, which represent sequences from the variable regions of cloned minicircles derived from a $\mathrm{Y}$ and a $\mathrm{Cl}$ strain [12]. As shown in Fig. 6A, the P173 probe 


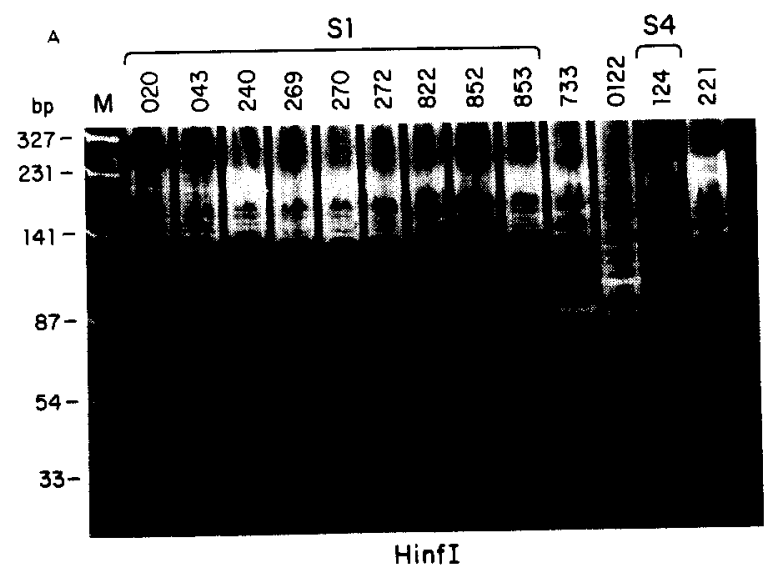

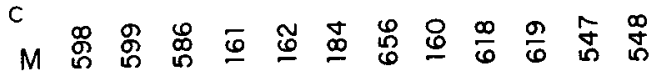

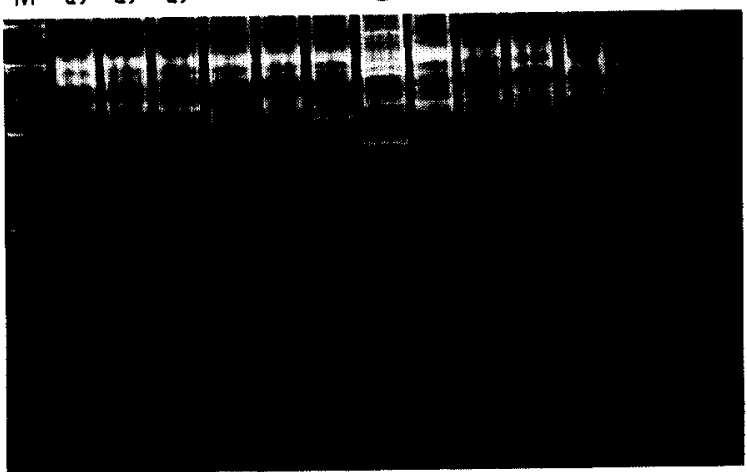

Hinf I

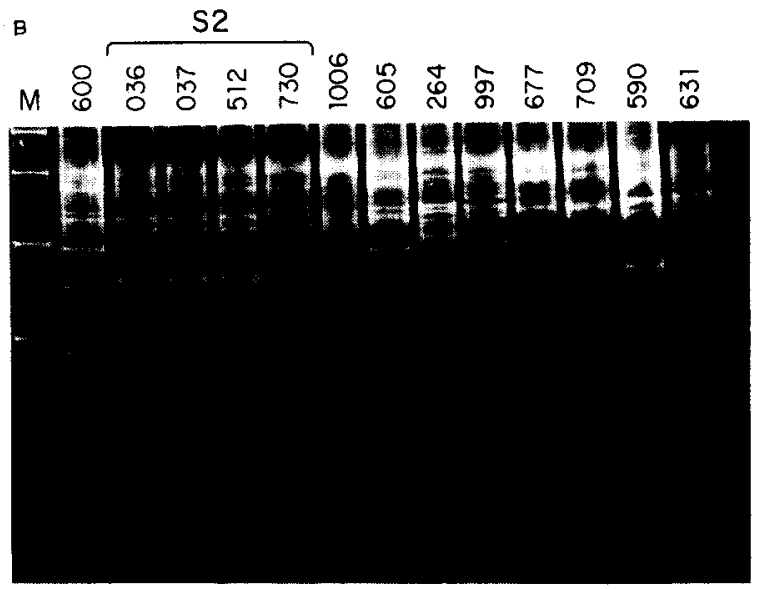

Hinf I

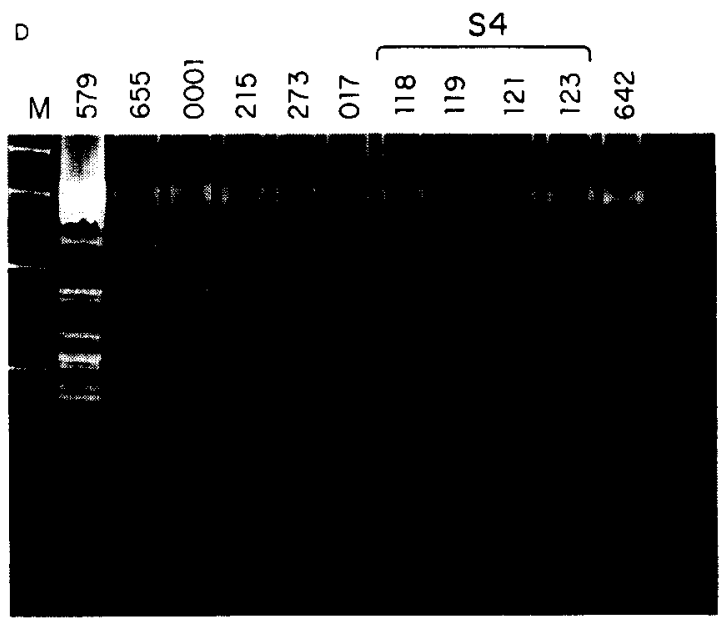

Hinf I

Fig. 5. Schizodeme analysis of $T$. cruzi stocks by restriction digestion of PCR-amplified minicircle variable region DNA. The 330-bp PCR products from the stocks listed in Table I were digested with Hinfl. Conditions same as in Fig. 3. Lane M, $\phi \times 174 / T a q I$ size marker.

hybridized with DNA from the actual cells from which the sequence was derived (2010) and also with DNA from two other samples which had been labeled as $Y$ strains $(3010,2011)$ and a fourth sample obtained from a Venezuelan bat (0001). These four samples belong to the S6 schizodeme. The probe did not hybridize to sample 579, which had also been labeled as a 'Y strain' by the contributing laboratory. The P174 probe hybridized with seven other samples belonging to the S2 schizodeme (2020, 3020, 036, $037,512,215$ and 730 ). No hybridization was detected with DNA from sample 4020 , which had been labeled as a ' $\mathrm{Cl}$ strain' by the contributing laboratory. Both filters were rewashed at $65^{\circ} \mathrm{C}$ (high stringency, $\mathrm{T}_{\mathrm{M}}-4^{\circ} \mathrm{C}$ ). The four $\mathrm{Y}$-strain specific hybrids with the $\mathrm{P} 173$ probe were stable even at this wash temperature (data not shown). The filter which was hybridized with the $\mathrm{Cl}$-strain probe (P174) showed a loss of signal from sample 215 when washed at a high stringency (Fig. 6B), indicating substantial mismatch between the probe and that particular minicircle sequence.

To correlate the above hybridization results with restriction enzyme patterns, the amplified variable region DNAs were digested with $R s a I$ and the 
A

PROBE $\begin{array}{lllllllllllll}1 & 2 & 3 & 4 & 5 & 6 & 7 & 8 & 9 & 10 & 11 & 12\end{array}$
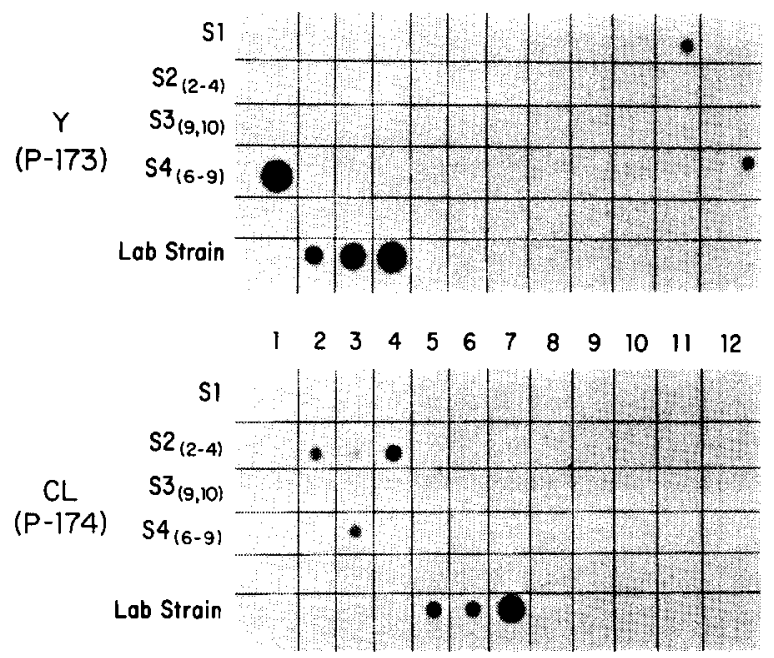

\begin{tabular}{|c|c|c|c|c|c|c|c|c|c|c|c|c|}
\hline & 1 & 2 & 3 & 4 & 5 & 6 & 7 & 8 & 9 & 10 & 11 & 12 \\
\hline S1 & $0^{33^{3}}$ & 8 & & $3^{2}$ & $2^{2}$ & $8^{62}$ & 82 & $2^{\circ}$ & $x^{\circ}$ & & & \\
\hline$S 2_{(2-4)}$ & $8^{\circ}$ & $0^{\circ}$ & & $5^{2}$ & $0^{\circ}$ & $6^{\circ}$ & $2^{88}$ & $0 \hat{9}$ & $6^{\hat{1}}$ & $p^{\circ}$ & $\mathscr{5}^{\circ}$ & $6^{3}$ \\
\hline$S 3_{(9,10)}$ & $9^{\circ}$ & $5^{\infty}$ & $5^{80}$ & 16 & $6^{2}$ & $8^{x}$ & $6^{60}$ & $60^{\circ}$ & $6^{8}$ & $6^{9}$ & $5^{x}$ & $5^{20}$ \\
\hline$S 4_{(6-9)}$ & $\rho^{\circ}$ & $6^{5}$ & & $\hat{\imath}^{3}$ & $0^{n}$ & 2 & $1^{8}$ & $2^{3}$ & $v^{9}$ & & & \\
\hline & $0^{2^{2}}$ & $\hat{3}^{3}$ & & $2^{2}$ & $n^{2}$ & & & & & & & \\
\hline & $5^{99}$ & $-\infty^{\circ}$ & & $e^{\circ}$ & 10 & $x^{6}$ & $x^{\infty}$ & $0^{20}$ & $60^{\circ}$ & & & \\
\hline
\end{tabular}

$B$

PROBE
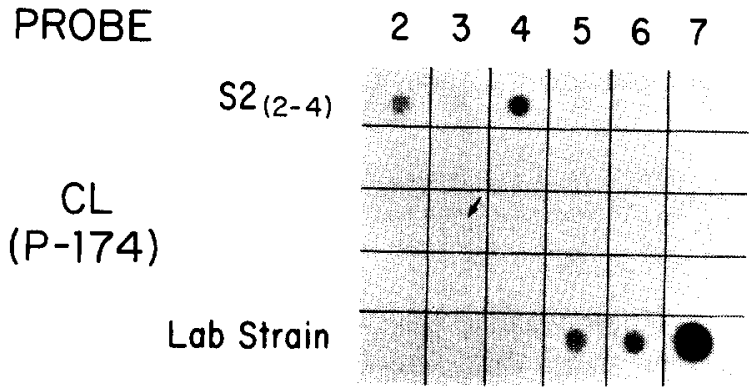

Fig. 6. Dot blot hybridizations of the amplified variable region DNAs with $\mathrm{Y}$ and $\mathrm{Cl}$ variable region oligonucleotide probes. (A) The 330-bp PCR-amplified DNAs from the $56 T$. cruzi stocks were spotted onto Nytran filters which were hybridized with $\mathrm{Y}$ (P173) or $\mathrm{Cl}$ (P174) variable region oligonucleotide probes. Washed at low stringency $\left(55^{\circ} \mathrm{C}, \mathrm{T}_{\mathrm{M}}-14^{\circ} \mathrm{C}\right)$. Subscripts by the schizodeme ' $S$ ' numbers denote the positions on the blot of the samples belonging to that particular schizodeme. Diagram below blots indicates the position of each of the 56 samples. (B) The same filter hybridized with the P174 oligonucleotide probe was rewashed at high stringency $\left(65^{\circ} \mathrm{C}\right.$, $\mathrm{T}_{\mathrm{M}}-4^{\circ} \mathrm{C}$ ). Arrow indicates sample 215 which gives no signal when rewashed at high stringency. acrylamide patterns compared, as shown in Fig. 7. This gel was blotted and the filter hybridized with the P173 probe, exposed, and then rehybridized with the P174 probe. The lanes on the right side of the blot in Fig. 7 were obtained from the initial hybridization with the $\mathrm{Y}$ probe, whereas the
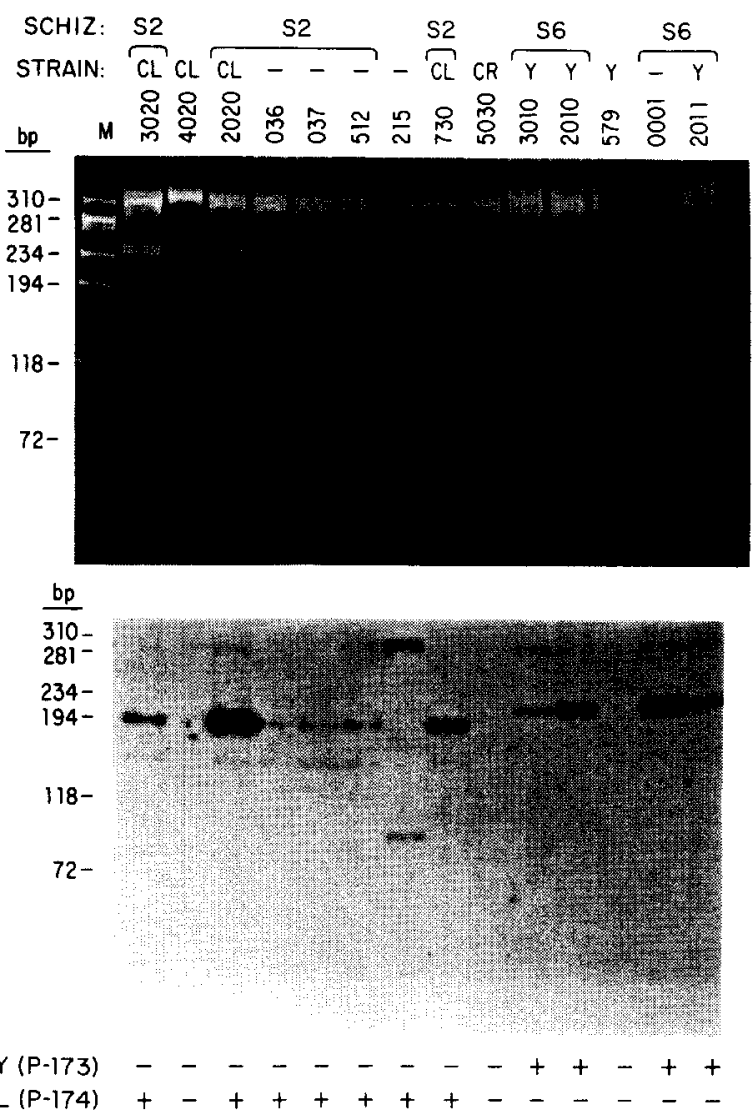

Fig. 7. Schizodeme analysis of the PCR-amplified variable region DNA from the putative $\mathrm{Y}$ and $\mathrm{Cl}$ strains. The amplified DNAs were digested with $R s a I$ and the restriction fragments separated in a 5-15\% acrylamide gel. The gel was stained with ethidium bromide and then electroblotted onto Nytran filter. The blot was hybridized first with the P173 probe and then rehybridized with the $\mathrm{P} 174$ probe. The filter was washed at low stringency $\left(55^{\circ} \mathrm{C}\right)$. Only the final exposure is shown, and the lanes which have bands hybridizing with either the $\mathrm{Y}$ or $\mathrm{Cl}$ probe are indicated by + or - . The autoradiograph lanes are aligned beneath the stained lanes. The stained sample lane 4020 was reproduced at a different exposure to better visualize the fragments. The putative strain classification of the samples is denoted above. Non-laboratory stocks are indicated by a - sign. Schizodeme analysis using the restriction fingerprints and the variable region oligomer probes agree, giving the same groupings. Schizodeme groups are denoted by the schizodeme ' $S$ ' number. Marker lane $\mathrm{M}$ is $\phi \mathrm{X} 174 /$ HaeIII DNA. 
lanes on the left side of the filter were obtained from the re-hybridization with the $\mathrm{Cl}$ probe. In the ethidium-stained gel, the relative homogeneity of the patterns from the $3020,2020,036,037$ and 512 stocks, which belonged to the $\mathrm{Cl}$ or $\mathrm{S} 2$ schizodeme, confirmed the close relatedness of these samples. The gel pattern of sample 215 , which showed a faint hybridization with the $\mathrm{Cl}$ probe at low stringency (Fig. 6A) and none at high stringency (Fig. 6B), showed no similarity to the others, and the gel pattern of sample 4020 , which showed no hybridization with the $\mathrm{P} 174$ probe even at low stringency, was also quite different from the others. The absence of hybridization of the P173 probe to sample 579 is shown, as is the absence of hybridization of the P174 probe to sample 4020 . The cross-hybridizing $R s a$ I DNA band from sample 215 with probe $P 173$ was larger than the major cross-hybridizing band in the other six samples, suggesting a restriction site polymorphism.

\section{Discussion}

Current methods for detection and classification of $T$. cruzi (xenodiagnosis, isoenzyme analysis, schizodeme analysis of total $\mathrm{kDNA}$ ) all require a cell amplification step in order to obtain enough material, thus possibly leading to selection of particular parasite strains in a mixed infection. The PCR schizodeme method overcomes this limitation by direct analysis of small quantities of parasite kinetoplast minicircle DNA obtained without cell outgrowth from a small sample of host blood or tissue [7].

Schizodeme analysis in general is dependent on the differential frequencies of kDNA minicircle sequence classes (defined by the variable region sequences) within a single network, and on the rapid rate of change of minicircle sequences in nature, leading to major differences between strains, especially in the case of $T$. cruzi [3]. The precise mechanism for the generation of minicircle sequence diversity is not well understood, and probably involves point mutations and recombination between different circles, as well as a possible selection of different minicircle sequence classes in the multiple clonal lines present in the $T$. cruzi parasite natural population [3,4]. 'Schizodeme' is an operational term for a group of parasites that are clearly genotypically related [2] and makes no assumptions about the cause of this relatedness. As such, schizodeme is a useful term for the operational classification of these parasites.

Whatever the mechanism for the generation of minicircle sequence diversity, this molecule has been shown to be a suitable genotypic marker for both species and strain of several kinetoplastids [14-16]. In addition to a variable region sequence that changes rapidly, the kinetoplastid minicircle contains another region, the sequence of which is fairly well conserved across the species. Several short subsequences within this conserved region are actually conserved across the kinetoplastid family, and are thought to be involved in the initiation of DNA replication $[17,18]$. In the case of $T$. cruzi, the conserved region is present as four conserved mini-repeats situated at $90^{\circ}$ from each other. We have shown previously that these minirepeats provide suitable species-specific primer sequences for amplification of the adjacent 330 bp variable region by the PCR method [7], and that the amplified variable region DNA represents the entire population of minicircle DNA. We have proposed to use this as a sensitive method for the diagnosis and classification of $T$. cruzi.

In this paper we have verified the generality of the PCR minicircle DNA amplification method for 56 strains and stocks of $T$. cruzi from widely separated geographical areas, and have also shown that schizodeme-specific variable region hybridization probes can be incorporated into this analysis method in addition to visualization of the acrylamide gel 'fingerprints'.

Due to the extreme heterogeneity of $T$. cruzi in nature, the schizodeme method is mainly useful for determination of closely related strains and for fingerprinting individual strains. We have limited the use of the term, schizodeme, to homogeneous subgroups of related strains, although more extended and more heterogeneous groupings can be observed with comparisons of total kDNA digestion profiles. It is possible that a more quantitative analysis of the digestion patterns, involving scoring the relative mobility and molar yield of each fragment and employing a statistical analysis of correlation coefficients may extend the range of this method.

A comparison of the schizodeme groupings 
determined by acrylamide gel analysis of total kDNA or the amplified variable region minicircle DNA shows a good correspondence between both methods. However the digestion patterns obtained from the amplified variable region minicircle DNA appear to be richer in information than those obtained from total kDNA, allowing for the classification of strains into additional schizodemes.

It is interesting that the samples in each schizodeme group are derived from the same geographical areas in each case. The only apparent exception to this rule is the 0001 stock, obtained from a bat in Venezuela, which we included in the S6 schizodeme, the original member of which was isolated in Brazil. However, a laboratory strain mixup is always a possibility.

A single geographical area can contain more than one schizodeme group, as seen in the case of the strains from the Bambui area, which comprise at least three different schizodemes $(S 1, S 2$, and S4). A number of samples were not classified into schizodeme groups and may represent the sole representatives of multiple schizodemes from different geographical areas. Analysis of additional $T$. cruzi isolates from the same geographical areas should permit the formation of additional schizodemes and allow the determination of the geographical range of each individual schizodeme, which is an important epidemiological consideration. The sensitivity of detection combined with the high degree of distinction of different strains of $T$. cruzi, using PCR minicircle DNA amplification, should permit the study of patterns of transmission of this parasite within and between geographical localities.

Hybridization of the amplified variable region DNA with schizodeme-specific oligonucleotide probes is a faster and more practical way for classifying $T$. cruzi into different schizodeme groups than analysis of acrylamide gel profiles of DNA digests. Our results indicate that for at least two schizodemes ( $\mathrm{S} 2$ and $\mathrm{S} 6$ ), there is a good correlation between the mobility of restriction enzyme fragments and the sequences of the fragments. The P173 probe hybridized with several samples which were labeled as $Y$ strain, but did not hybridize with sample 579, which was also labeled as a $\mathrm{Y}$ strain. We attribute this to strain mixup, and point out that this demonstrates the power of this method. The relatively stronger hybridization to the 0001 sample derived from a Venezuelan bat is probably due to a higher abundance of that particular minicircle sequence class in that strain than in the homologous 2010 strain. Likewise, the P174 probe hybridizes with several samples that were labeled as $\mathrm{Cl}$ strains, but does not hybridize with the 4020 sample, which was also labeled as $\mathrm{Cl}$ strain. The likelihood of a laboratory strain mixup in this case is made plausible by the completely different restriction gel pattern for this sample. However, the hybridization of the P174 probe to the 215 sample, which has a different Rsa gel pattern than the $\mathrm{Cl}$ samples, might appear to indicate that this variable region probe is not schizodeme-specific. However, the fact that the hybridization vanished at high wash stringency indicates that there is substantial mismatch, and the different sizes of the hybridizing fragments indicates the presence of restriction site polymorphisms. Therefore, the two variable region probes used in this study appear to be schizodeme-specific based on the 56 samples analyzed. However, additional work is necessary to test the general hypothesis that specific variable region probes can be generated for every $T$. cruzi schizodeme.

The availability of schizodeme-specific probes would allow an analysis to be performed using the oligonucleotide bound to a solid state medium such as a microtiter well or a membrane filter $[19,20]$. A PCR-amplified unknown minicircle variable region sample could be tested against a library of such specific variable region probes by a single hybridization, and the results analyzed using non-radioactive technology. We suggest that this method should prove useful in the rapid and sensitive diagnosis of $T$. cruzi parasites in chronic Chagasic patients and in blood banks, as well as in epidemiological studies.

\section{Acknowledgements}

This work received financial support from the UNDP/World Bank/WHO Special Programme for Research and Training in Tropical Diseases and from a WHO/Rockefeller Foundation bilateral research grant. H. A. was supported by a Gradu- 
ate Opportunity Fellowship and an NIH predoctoral Training Grant (AI 07323). We would like to thank Nancy Sturm and Wim Degrave for helpful discussions.

\section{References}

1 Miles, M., Souza, A., Povoa, M., Shaw, J., Lainson, R. and Toye, P. (1978) Isozymic heterogeneity of Trypanosoma cruzi in the first autochthonous patients with Chagas' disease in Amazonian Brazil. Nature 272, 819-821.

2 Morel, C., Chiari, E., Camargo, E., Mattei, D., Romanha, A. and Simpson, L. (1980) Strains and clones of Trypanosoma cruzi can be characterized by restriction endonuclease fingerprinting of kinetoplast DNA minicircles. Proc. Natl. Acad. Sci. USA 77, 6810-6814.

3 Tibayrenc, M., Ward, P., Moya, A. and Ayala, F. (1986) Natural populations of Trypanosoma cruzi, the agent of Chagas' disease, have a complex multiclonal structure. Proc. Natl. Acad. Sci. USA 83, 115-119.

4 Tibayrenc, M. and Ayala, F. (1988) Isoenzyme variability in Trypanosoma cruzi, the agent of Chagas' disease: Genetical, taxonomic and epidemiological significance. Evolution 42, 277-292.

5 Goncalves, A., Chiari, E., Carneiro, M., Romanha, A. and Morel, C. (1984) Schizodeme characterization of natural and artificial populations of Trypanosoma cruzi as a tool in the study of Chagas' disease. In: Application of Biochemical and Molecular Biology Techniques to Problems of Parasite and Vector Identification. (Newton, B. and Michal, I., eds.), pp. 253-274, UNDP/WHO Special Programme for Research and Training in Tropical Diseases, Geneva, Switzerland.

6 Deane, M., Sousa, M., Pereira, N., Goncalves, A., Momen, H. and Morel, C. (1984) Trypanosoma cruzi: inoculation schedules and re-isolation methods select individual strains from doubly infected mice, as demonstrated by schizodeme and zymodeme analyses. J. Protozool. 31, 276-280.

7 Sturm, N., Degrave, W., Morel, C. and Simpson, L. (1989) Sensitive detection and schizodeme classification of Trypanosoma cruzi cells by amplification of kinetoplast minicircle DNA sequences: use in diagnosis of Chagas' disease. Mol. Biochem. Parasitol. 33, 205-214.

8 Gomez-Eichelmann, M.C., Holz, Jr., G., Beach, D., Simpson, A.M. and Simpson, L. (1988) Comparison of several lizard Leishmania species and strains in terms of kinetoplast minicircle and maxicircle DNA sequences, nuclear chromosomes, and membrane lipids. Mol. Biochem. Parasitol. 27, 143-158.
9 Goncalves, A., Nehme, N. and Morel, C. (1984) Trypanosomatid characterization by schizodeme analysis. In: Genes and Antigens of Parasites. A Laboratory Manual. (Morel, C.M., ed.), pp. 95-109, Oswaldo Cruz Foundation, Rio de Janeiro.

10 Goncalves, A., Nehme, N.S. and Morel, C. (1990) An improved silver staining procedure for schizodeme analysis in polyacrylamide gradient gels. Mem. Inst. Oswaldo Cruz 85, 101-106.

11 Brunk, C. and Simpson, L. (1977) Comparison of various ultraviolet sources for fluorescent detection of ethidium bromide-DNA complexes in polyacrylamide gels. Anal. Biochem. 82, 455-462.

12 Degrave, W., Fragoso, S., Britto, C., Van Heuverswyn, H., Kidane, G., Cardoso, M., Mueller, R., Simpson, L. and Morel, C. (1988) Peculiar sequence organization of kinetoplast minicircles from Trypanosoma cruzi. Mol. Biochem. Parasitol. 27, 63-70.

13 Wood, W., Gitschier, J., Lasky, L. and Lawn, R. (1985) Base composition-independent hybridization in tetramethylammonium chloride: A method for oligonucleotide screening of highly complex gene libraries. Proc. Natl. Acad. Sci. USA 82, 1585-1588.

14 Frasch, A., Goijman, S., Cazzulo, J. and Stopanni, A. (1981) Constant and variable regions in DNA minicircles from Trypanosoma cruzi and Trypanosoma rangeli: Application to species and stock differentiation. Mol. Biochem. Parasitol. 4, 163-170.

15 Spithill, T. and Grumont, R. (1984) Identification of species, strains and clones of Leishmania by characterization of kinetoplast DNA minicircles. Mol. Biochem. Parasitol. 12, 217-236.

16 Lopes, U. and Wirth, D. (1986) Identification of visceral Leishmania species with cloned sequences of kinetoplast DNA. Mol. Biochem. Parasitol. 20, 77-84.

17 Ntambi, J. and Englund, P. (1985) A gap at a unique location in newly replicated kinetoplast DNA minicircles from Trypanosoma equiperdum. J. Biol. Chem. 260, 5574-5579.

18 Simpson, L. (1987) The mitochondrial genome of kinetoplastid protozoa: Genomic organization, transcription, replication and evolution. Annu. Rev. Microbiol. 41, 363-382.

19 Kemp, D., Smith, D., Foote, S., Samaras, N. and Peterson, G. (1989) Colorimetric detection of specific DNA segments amplified by polymerase chain reactions. Proc. Natl. Acad. Sci. USA 86, 2423-2427.

20 Saiki, R., Walsh, P., Levenson, C. and Erlich, H. (1989) Genetic analysis of amplified DNA with immobilized sequence-specific oligonucleotide probes. Proc. Natl. Acad. Sci. USA 86, 6230-6234. 